\title{
Sugar palm starch biopolymer: extraction and processing
}

\begin{abstract}
Chapter 3 describes the extraction and processing of sugar palm starch (SPS) biopolymer. A systematic step for extracting the starch using an alkaline method followed by hydrolysis using protease is explained. A modified method using antimicrobial agents is also presented which provides shorter soaking times with less enzyme and shorter centrifugation time during extraction. It was found that the process of extracting starch from the stem of the sugar palm is the same as the process of extracting commercially available starch from the sago palm. It was postulated that SPS can behave like thermoplastic starch in the presence of glycerol whereby glycerol has been widely used as plasticizer due to its good behavior as an additive to the starch.
\end{abstract}

